\title{
Osteopontin, Macrophage Migration Inhibitory Factor and Anti-Interleukin-8 Autoantibodies Complement CA125 for Detection of Early Stage Ovarian Cancer
}

\author{
Jing Guo ${ }^{1,2}$, Wei-Lei Yang ${ }^{2}{ }^{(0)}$, Daewoo Pak ${ }^{3}{ }^{(0)}$, Joseph Celestino ${ }^{4}$, Karen H. Lu ${ }^{4}$, Jing Ning ${ }^{3}$, \\ Anna E. Lokshin ${ }^{5}$, Zhongping Cheng ${ }^{1}$, Zhen Lu ${ }^{2, *}$ and Robert C. Bast Jr. ${ }^{2, *}$ \\ 1 Department of Obstetrics and Gynecology, Shanghai Tenth People's Hospital, Tongji University School of \\ Medicine, Shanghai 200072, China; camelguo2012@gmail.com (J.G.); mdcheng18@tongji.edu.cn (Z.C.) \\ 2 Department of Experimental Therapeutics, University of Texas M.D. Anderson Cancer Center, Houston, \\ TX 77030, USA; newworldyang@gmail.com \\ 3 Department of Biostatistics, University of Texas M.D. Anderson Cancer Center, Houston, TX 77030, USA; \\ DPak@mdanderson.org (D.P.); jning@mdanderson.org (J.N.) \\ 4 Department of Gynecologic Oncology and Reproductive Medicine, University of Texas M.D. Anderson \\ Cancer Center, Houston, TX 77030, USA; jcelesti@mdanderson.org (J.C.); khlu@mdanderson.org (K.H.L.) \\ 5 Department of Epidemiology, Pathology, Medicine, and Obstetrics/Gynecology and Reproductive Sciences, \\ University of Pittsburgh, Pittsburgh, PA 15260, USA; loksax@UPMC.EDU \\ * Correspondence: zlu@mdanderson.org (Z.L.); rbast@mdanderson.org (R.C.B.J.); \\ Tel.: +1-713-563-3721 (Z.L.); +1-713-792-7743 (R.C.B.J.)
}

Received: 1 April 2019; Accepted: 24 April 2019; Published: 28 April 2019

\begin{abstract}
Early detection of ovarian cancer promises to reduce mortality. While serum CA125 can detect more than $60 \%$ of patients with early stage (I-II) disease, greater sensitivity might be observed with a panel of biomarkers. Ten protein antigens and 12 autoantibody biomarkers were measured in sera from 76 patients with early stage (I-II), 44 patients with late stage (III-IV) ovarian cancer and 200 healthy participants in the normal risk ovarian cancer screening study. A four-biomarker panel (CA125, osteopontin (OPN), macrophage inhibitory factor (MIF), and anti-IL-8 autoantibodies) detected $82 \%$ of early stage cancers compared to $65 \%$ with CA125 alone. In early stage subjects the area under the receiver operating characteristic curve (AUC) for the panel (0.985) was significantly greater $(p<0.001)$ than the AUC for CA125 alone (0.885). Assaying an independent validation set of sera from 71 early stage ovarian cancer patients, 45 late stage patients and 131 healthy women, AUC in early stage disease was improved from 0.947 with CA125 alone to 0.974 with the four-biomarker panel $(p=0.015)$. Consequently, OPN, MIF and IL-8 autoantibodies can be used in combination with CA125 to distinguish ovarian cancer patients from healthy controls with high sensitivity. Osteopontin appears to be a robust biomarker that deserves further evaluation in combination with CA125.
\end{abstract}

Keywords: ovarian cancer; osteopontin (OPN); macrophage migration inhibitory factor (MIF); CA125; IL-8 autoantibodies (IL-8 AAb); early detection

\section{Introduction}

Ovarian cancer is diagnosed at late stage (III, IV) in more than 70\% of cases, contributing to poor patient outcomes. Over 22,530 women in the US will be diagnosed with ovarian cancer this year and about 13,980 women will die from the disease [1]. While five-year survival has improved over the last three decades, long term survival has changed little over the same interval and less than $30 \%$ for patients with late stage disease can be cured [2]. By contrast, when ovarian cancer is diagnosed in stage I, up to $90 \%$ of patients can be cured with conventional surgery and chemotherapy. Even in Stage 
II, up to $70 \%$ of patients survive five years with currently available therapy [2]. Consequently, survival might be significantly improved by detecting early stage ovarian cancer in a larger fraction of patients.

Attempts to detect ovarian cancer have utilized serum biomarkers, notably CA125, and imaging with transvaginal sonography (TVS). Used individually, neither CA125 nor TVS is sufficiently sensitive or specific for cost-effective screening [3]. Two-stage strategies have proven more promising, where rising CA125, judged by the risk of ovarian cancer algorithm (ROCA), has triggered TVS in a small fraction of participants, prompting surgery when imaging suggests possible cancer. Two clinical screening trials-the normal risk ovarian cancer screening study (NROSS) [4] and the United Kingdom Collaborative Study of Ovarian Cancer Screening (UKCTOCS) [5] - have achieved adequate specificity with three to four operations for each case of ovarian cancer detected. Both trials have detected a larger fraction of early stage (I-II) cases (40-64\%) than observed at the time of conventional diagnosis (20-25\%). With 200,000 participants, the UKCTOCS was powered to detect a survival advantage and in one pre-specified subset of women screened for seven years a $20 \%$ reduction in mortality was observed, although additional follow-up will be required to assure the accuracy of this estimate.

Whatever the outcome of the UKCTOCS, there is room for improvement in both phases of the two-stage strategy. CA125 is expressed by only $80 \%$ of ovarian cancers and is elevated in sera from approximately $65 \%$ of patients with early stage disease. Additional biomarkers will be required to detect a larger fraction of cases. Several serum biomarkers have shown promise for early detection in blood samples from ovarian cancer patients at the time of clinical diagnosis [4-8]. Both HE4 and CA72.4 can detect up to $16 \%$ of cases missed by CA125 [9].

While serum proteins have been studied extensively [10], most biomarkers are also expressed by normal tissues and are found at low levels in sera from healthy women. Depending upon antigen expression in ovarian cancers and the rate of release into blood, a substantial volume of ovarian cancer may be required to shed sufficient amounts of protein antigen to elevate serum levels. Smaller volumes of cancer might, however, evoke an immune response resulting in the production of autoantibodies $(\mathrm{AAb})$ against ovarian cancer-associated antigens [11]. Virtually all high grade serous ovarian cancers have mutations of p53 with overexpression of TP53 protein in a majority of cases. Autoantibodies reactive with TP53 are associated with at least $20 \%$ of high grade serous ovarian cancers. Titers of anti-TP53 AAb rise eight months prior to CA125 and 22 months prior to clinical diagnosis of cancers that do not elevate serum CA125 levels [4].

The objective of this study is to evaluate 10 candidate antigens [6,12-21] and 12 potential $\mathrm{AAb}$ biomarkers $[5,6,22-28]$ that have been cited in the world literature for their ability to distinguish patents with early stage ovarian cancer from healthy individuals, seeking biomarkers that would detect cases missed by CA125. Serum specimens have been analyzed from patients with early stage (I-II) and late stage (III-IV) ovarian cancer and from healthy women who participated in the NROSS study. In both discovery and validation sets, a four-biomarker panel of osteopontin, macrophage migration inhibitory factor, IL-8 AAb and CA125 exhibited greater sensitivity than CA125 alone in detecting early stage ovarian cancer.

\section{Results}

2.1. Osteopontin (OPN), Macrophage Migration Inhibitory Factor (MIF) and Anti-IL-8 Autoantibodies (AAb) Provide the Highest Sensitivity at 98\% Specificity Among 22 Potential Ovarian Cancer Biomarkers

Using Luminex-based Magplex/xMAP assays [4], we measured levels of 10 ovarian cancer associated protein antigens (Osteopontin (OPN), Macrophage Migration Inhibiting Factor (MIF), Human Epididymis Protein 4 (HE4), Leptin, Interleukin-6 (IL-6), Interleukin-8 (IL-8), Tumor Necrosis Factor $\alpha(\mathrm{TNF} \alpha)$, Fibroblast Growth Factor 2 (FGF2), Transforming Growth Factor alpha (TGF $\alpha$ ), and Vascular Endothelial Growth Factor (VEGF) and 12 autoantibodies reactive with cancer-associated proteins (anti-IL-8, E3 ubiquitin-protein ligase Mdm2 (MDM2), Myc proto-oncogene protein (c-MYC), Tissue-type Plasminogen Activator (PLAT), Epithelial Cell Adhesion Molecule (EpCAM), Homeobox Protein Hox-A7 (HOXA7), Alpha-enolase (ENO1), Protein Disulfide-isomerase (PDI), Endoplasmic 
Reticulum Chaperone BiP (HSPA5), Heat Shock Cognate $71 \mathrm{kDa}$ Protein (HSPA8), Annexin A2 (ANXA2), and cathepsin D) in sera from 76 patients with early stage (I-II) ovarian cancer, 44 patients with late stage (III-IV) ovarian cancer, and 200 healthy controls who participated in the NROSS study. Characteristics of the cancers are listed in Table 1.

Table 1. Characteristics of the patient population.

\begin{tabular}{|c|c|c|c|c|c|c|c|c|c|}
\hline Sample set & Stage & No. & \multicolumn{5}{|c|}{ Histology } & \multicolumn{2}{|c|}{ Age (Years) } \\
\hline \multirow{4}{*}{$\begin{array}{c}\text { Discovery } \\
\text { set }\end{array}$} & I & 50 & 15 & 13 & 7 & 7 & 8 & 56 & 55 \\
\hline & III & 34 & 33 & 0 & 1 & 0 & 0 & 63 & 61 \\
\hline & IV & 10 & 9 & 0 & 0 & 0 & 1 & 60 & 58 \\
\hline & Healthy & 200 & & & & & & 62 & 63 \\
\hline \multirow{4}{*}{$\begin{array}{c}\text { Discovery } \\
\text { set }\end{array}$} & II & 30 & 23 & 2 & 1 & 0 & 4 & 60 & 61 \\
\hline & III & 39 & 39 & 0 & 0 & 0 & 0 & 62 & 61 \\
\hline & IV & 6 & 6 & 0 & 0 & 0 & 0 & 54 & 59 \\
\hline & Healthy & 131 & & & & & & 66 & 66 \\
\hline
\end{tabular}

For cost-effective two-stage screening strategies, no more than $2 \%$ of patients with elevated serum biomarkers should be referred for imaging with TVS [29,30]. Consequently, we have measured the sensitivity of biomarker candidates at $98 \%$ specificity. Among the 22 biomarkers, the highest sensitivity was observed with OPN, MIF and anti-IL-8 AAb (Figures S1-S6, Table S1). OPN was elevated in 50\% of early stage and $46 \%$ of late stage cases (Figure 1A). Elevated MIF was detected in $21 \%$ of early stage and $39 \%$ of late stage ovarian cancers (Figure 1B). Anti-IL-8 autoantibodies were detected in $7 \%$ of both early and late stage cases (Figure 1C). CA125, measured with a Roche assay had the highest sensitivity, detecting $65 \%$ of early stage and $89 \%$ of late stage ovarian cancer cases with a cut-off of $35 \mathrm{U} / \mathrm{mL}$ (Figure 1D).

\subsection{OPN, MIF and Anti-IL-8 Autoantibody Levels Are Elevated in an Independent Validation Set}

OPN, MIF, anti-IL-8 AAb and CA125 levels were measured in an independent validation set of sera from 71 patients with early stage ovarian cancer, 45 patients with late stage ovarian cancer and 131 healthy participants in the NROSS trial. Patient characteristics are provided in Table 1. OPN was elevated in $20 \%$ of early stage and $16 \%$ of late stage disease (Figure $2 \mathrm{~A}$ ). MIF was elevated in $3 \%$ of early stage cases and 7\% of late stage cases (Figure 2B). Elevated anti-IL-8 AAb levels were detected $10 \%$ of sera from early stage and $9 \%$ from late stage cases (Figure 2C). CA125 was elevated in $78 \%$ of early and $98 \%$ of late stage cases (Figure 2D). When control values in the validation and discovery sets were compared, controls in the validation set exhibited higher levels of OPN and MIF, but similar levels of anti-IL-8 AAb, contributing to the decrease in sensitivity observed for OPN and MIF at $98 \%$ specificity. 

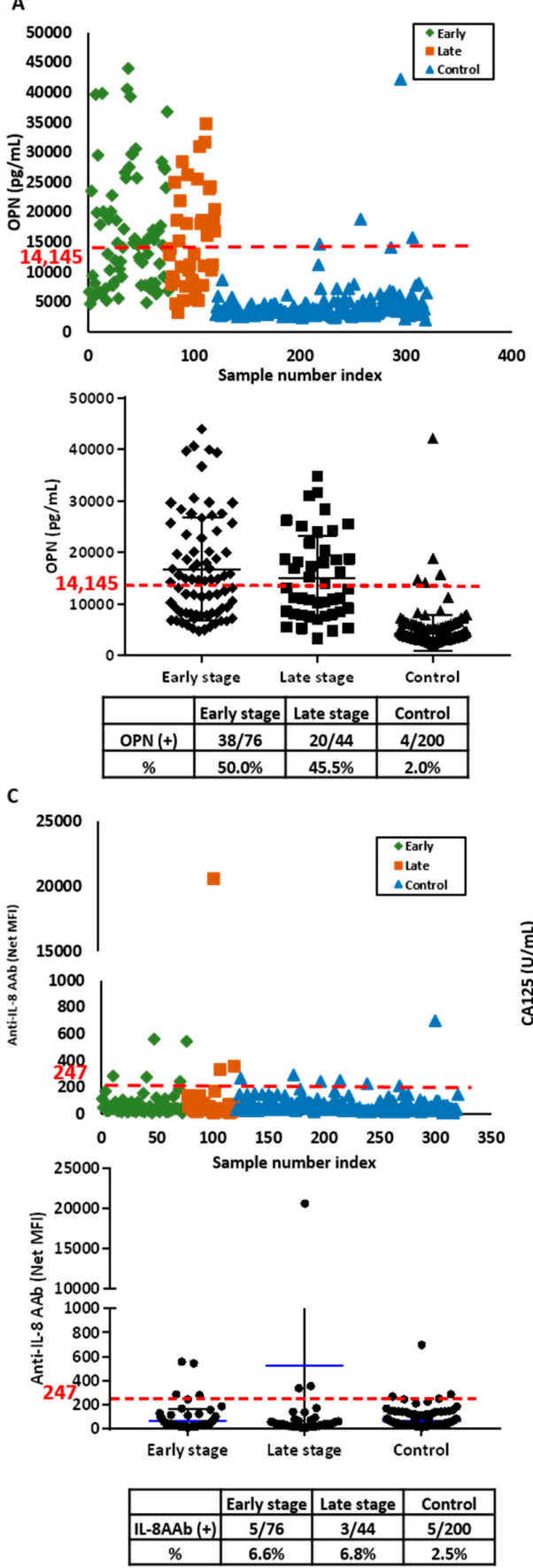
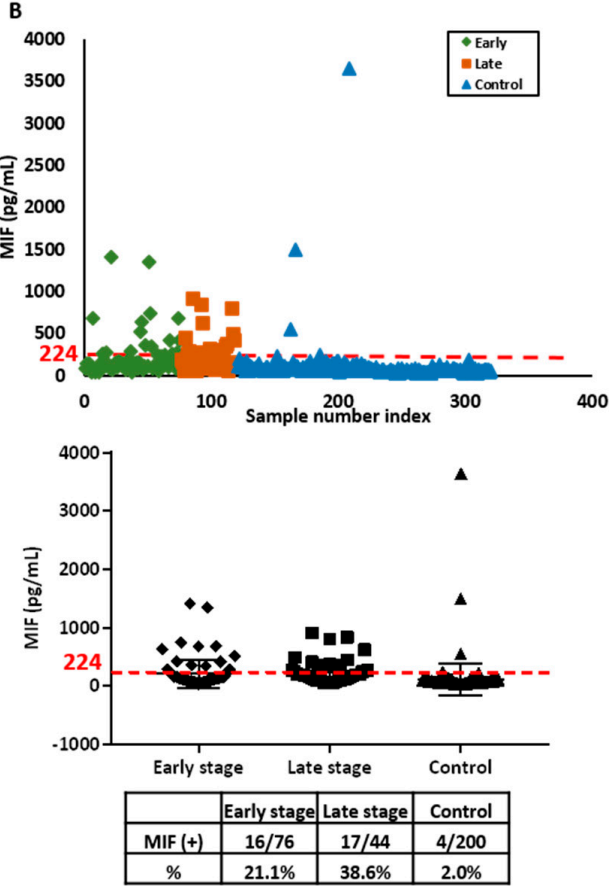

D
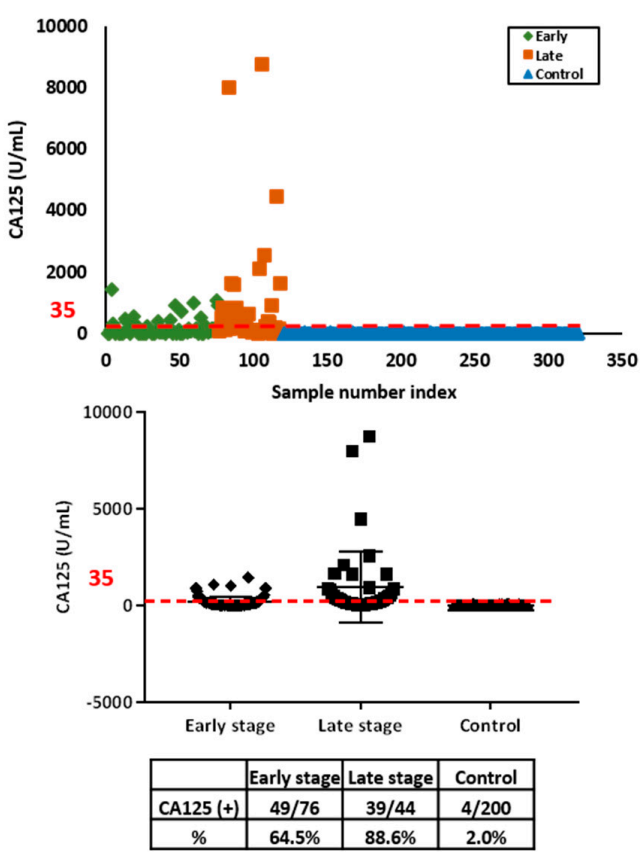

Figure 1. Serum levels of osteopontin (OPN), macrophage inhibitory factor (MIF) anti-IL-8 autoantibody and CA125 are elevated in the discovery set from ovarian cancer patients and healthy controls. (A) OPN antigen; (B) MIF antigen; (C) anti-IL-8 autoantibodies (AAb) and (D) CA125 antigen. In the upper panels, each symbol represents the average of duplicate serum samples from individual early stage ovarian cancer cases (green), late stage ovarian cancer cases (red) or controls (blue). The middle panels contain a scatter plot where the blue line represents the median value in early and late stage case and control groups, respectively. The red dashed lines in each plot represent the cut-off value at $98 \%$ specificity for OPN, MIF and anti-IL-8 AAb, and cut-off value of $35 \mathrm{U} / \mathrm{mL}$ for CA125. The lower panel is a table displaying the sensitivity for early and late stage ovarian cancer cases and controls near $98 \%$ specificity. 
A
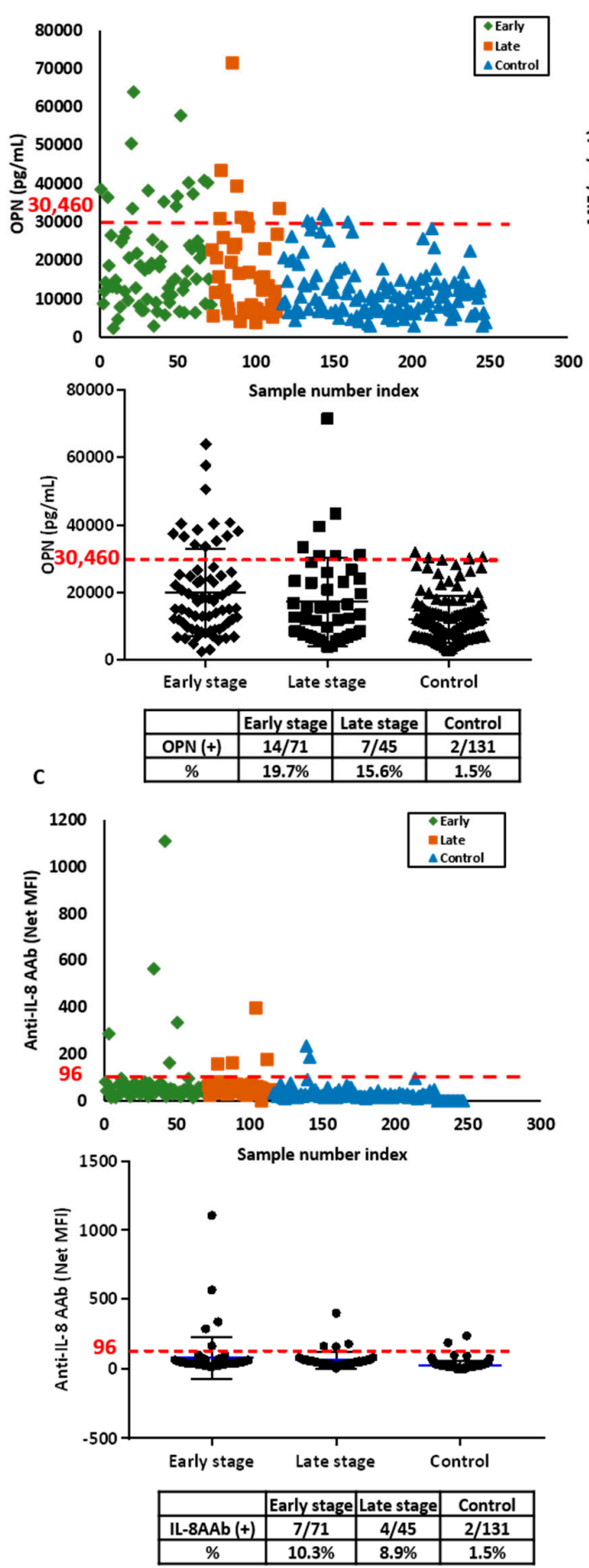

B
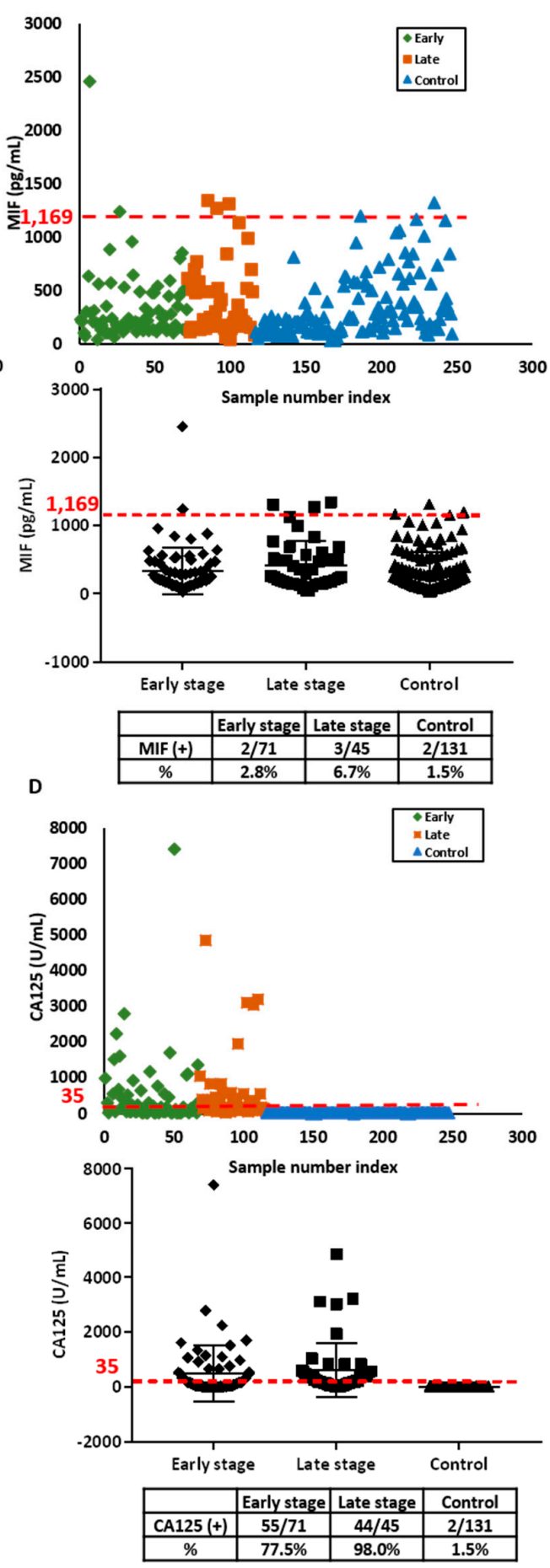

Figure 2. Serum levels of OPN, MIF anti-IL-8 autoantibody and CA125 are elevated in the validation set from ovarian cancer patients and healthy controls. (A) OPN antigen; (B) MIF antigen; (C) anti-IL-8 autoantibody and (D) CA125 antigen. In the upper panels, each symbol represents the average of duplicate serum samples from individual early stage ovarian cancer cases (green), late stage ovarian cancer cases (red) or controls (blue). The middle panels contain a scatter plot where the blue line represents the median value in early and late stage case and control groups, respectively. The red dashed lines in each plot represent the cut-off value at 98\% specificity for OPN, MIF and anti-IL-8 AAb, and cut-off value of $35 \mathrm{U} / \mathrm{mL}$ for CA125. The lower panel is a table displaying the sensitivity for early and late stage ovarian cancer cases and controls near $98 \%$ specificity. 


\subsection{OPN, MIF and IL-8 Autoantibody Complement CA125 for Detecting Ovarian Cancer}

Statistical tests based on bootstrap were used to compare AUCs and partial AUCs (pAUCs) to identify the most sensitive combination of biomarkers. Early stage cases had significantly different AUCs between different pairs of models (Table 2).

Table 2. Comparison of AUC and pAUC for biomarker models in the discovery set.

\begin{tabular}{cccc}
\hline Stage & Marker & AUC & pAUC \\
\hline \multirow{3}{*}{ Early stage } & CA125 & 0.885 & 0.013 \\
& CA125 MIF & $(0.833,0.937)$ & $(0.011,0.015)$ \\
& OPN & $(0.974,0.996)$ & $(0.012,0.018)$ \\
& CA125 MIF & $0.985 \#$ & 0.015 \\
& OPN IL8AAb & $(0.975,0.995)$ & $(0.012,0.018)$ \\
Late stage & CA125 & 0.969 & 0.018 \\
& CA125 MIF & $(0.931,1.000)$ & $(0.016,0.020)$ \\
& OPN & $(0.998,1.000)$ & $(0.018,0.020)$ \\
& CA125 MIF & 0.999 & 0.019 \\
& OPN IL8AAb & $(0.998,1.000)$ & $(0.017,0.020)$ \\
\hline
\end{tabular}

Compared to CA125 alone, ${ }^{*} p<0.001 ; \# p<0.001$.

The model that contained all four biomarkers CA125, OPN, MIF and anti-IL-8 AAb had the highest AUC $=0.985$ (95\% CI: 0.975-0.995) which differed significantly $(p<0.001)$ from the AUC $=0.885$ (95\% CI: 0.833-0.937) for CA125 alone. Late stage cases also had different AUC values with different combinations of biomarkers. A combination of CA125, OPN and MIF had the highest AUC = 0.999 (95\% CI: 0.998-1.000). When assessing the receiver operating characteristic (ROC) while combining all four markers, our AUC was 0.985 and 0.999 in early and late stage subjects respectively (Figure 3A,B). We then applied two models that had shown the best performances (model 1: CA125, OPN and MIF; model 2: CA125, OPN, MIF and IL-8AAb) to an independent validation data set for early and late stage samples. The validation models had similar AUC and pAUC (Figure 3C,D and Table 3). In early stage disease the four-biomarker combination had a significantly higher $(p=0.015)$ AUC $=0.974(95 \%$ CI 0.957-0.991) than the AUC $=0.947$ (95\% CI: 0.915-0.978) for CA125 alone. In the discovery set, addition of the three biomarkers improved detection of early stage cancers from 65\% with CA125 alone to $82 \%$. In the validation set, the addition of the three biomarkers also improved detection for early stage disease from $78 \%$ with CA125 alone to $85 \%$.

Table 3. Comparison of AUC and pAUC for biomarker models in the validation set.

\begin{tabular}{cccc}
\hline Stage & Marker & AUC & pAUC \\
\hline \multirow{4}{*}{ Early stage } & CA125 & 0.947 & 0.015 \\
& CA125 MIF & $(0.915,0.978)$ & $(0.012,0.017)$ \\
& OPN & $(0.927,0.983)$ & $(0.013,0.017)$ \\
& CA125 MIF & $0.974^{*}$ & 0.015 \\
& OPN IL8AAb & $(0.957,0.991)$ & $(0.013,0.017)$ \\
Late stage & CA125 & 0.999 & 0.999 \\
& CA125 MIF & $(0.997,1.000)$ & $(0.997,1.000)$ \\
& OPN & $(1.000,1.000)$ & $(1.000,1.000)$ \\
& CA125 MIF & 1.000 & 1.000 \\
& OPN IL8AAb & $(1.000,1.000)$ & $(1.000,1.000)$ \\
\hline
\end{tabular}

Compared to CA125 alone, ${ }^{*} p=0.0153$. 
A

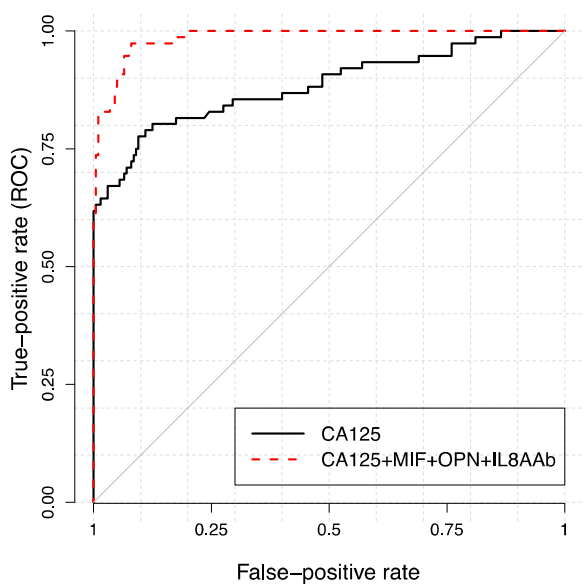

C

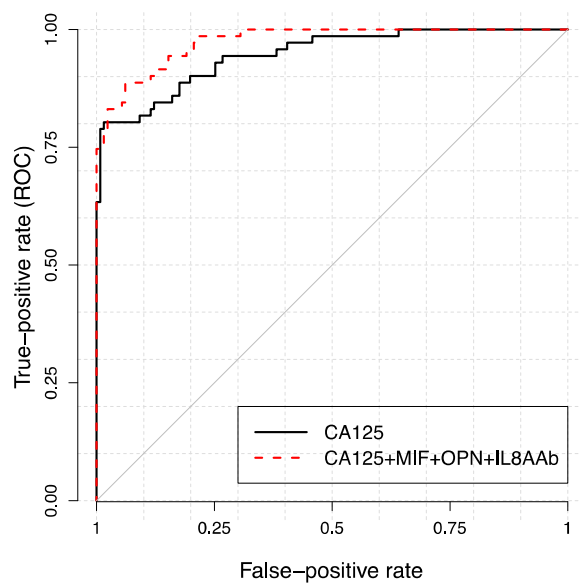

B

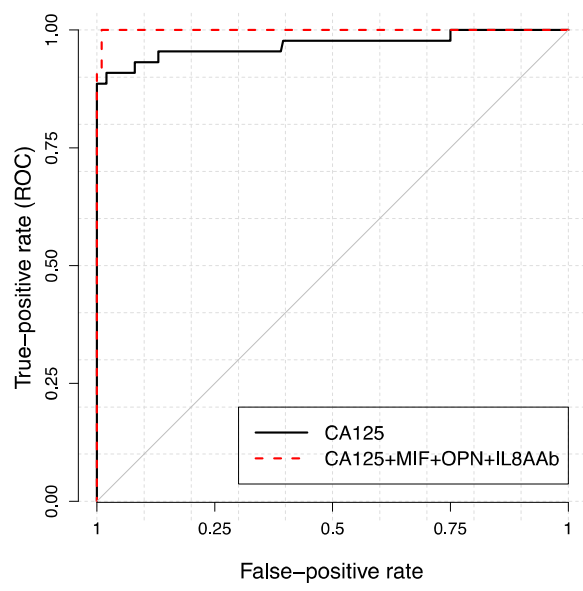

D

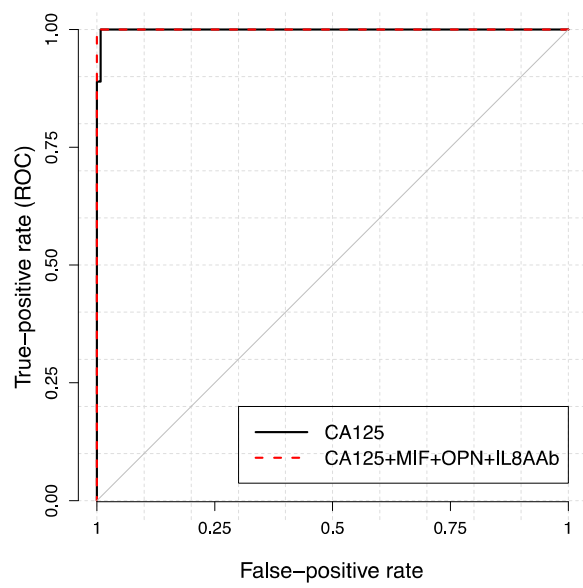

Figure 3. Receiver operating characteristic (ROC) curves for a combination of CA125, OPN, MIF and anti-IL-8 autoantibody biomarkers. (A) Early stage cases of discovery set. (B) Late stage cases of discovery set. (C) Early stage cases of validation set. (D) Late stage cases of validation set. A combination of CA125, OPN, MIF and IL-8 AAb levels had a significantly greater AUC (0.985) than did CA125 alone in early stage cases of discovery set (AUC $=0.885, p<0.001$ ). Compare to CA125 alone (AUC $=0.947$ ), the combination also made a significant difference (AUC $=0.974, p=0.015$ ) in early stage cases of validation set.

\section{Discussion}

In this study we have identified three biomarkers-OPN, MIF and anti-IL-8 AAb-that detect early stage ovarian cancers missed by CA125. Addition of these three biomarkers to CA125 achieved a sensitivity of $82-85 \%$ for detecting early stage ovarian cancer compared to $65-78 \%$ with CA125 alone in two independent data sets. While OPN and MIF have previously been cited as potential biomarkers for ovarian cancer [31,32], in this study we have had the opportunity for the first time to evaluate OPN and MIF in large numbers of patients with early stage disease.

Complementarity between CA125 and OPN, MIF and anti-IL-8 AAb was demonstrated by ROC analysis of both the discovery set and the validation sets of sera from patients with early stage ovarian cancer. The added values of the three biomarkers were most readily apparent in the discovery set where CA125 detected $65 \%$ of the cases and the four-biomarker panel $82 \%$. In the validation set, CA125 alone detected 78\% of early stage cases, a much higher fraction than anticipated, decreasing the apparent impact of other biomarkers. Given the greater sensitivity of CA125 for the serous histotype of epithelial ovarian cancer, detection of a larger fraction of early stage cancers with CA125 alone may relate to the larger fraction of serous cancers in the validation set (59\%) than in the discovery set 
(36\%). Use of the four biomarkers increased detection in the validation set to $85 \%$, but the greatest contribution was provided by OPN.

OPN is a glycophosphoprotein that is secreted into body fluids by normal osteoblasts, arterial smooth muscle cells, different epithelial cells, activated $\mathrm{T}$ cells and macrophages, but can also be overexpressed by cancers that arise at different sites, including ovarian cancer [33,34]. Functionally, OPN can promote ovarian cancer growth in cell culture and in vivo by activating the PI3K/AKT/HIF-1 $\alpha$ signaling pathway; OPN expression positively correlates with clinical stage, histological grade and lymph node metastasis [35-37]. In earlier studies, we have shown that OPN can be detected in CA125 negative ovarian cancers at the level of tissue expression, consistent with our observations with circulating OPN in this report [32].

MIF was one of the first cytokines to be described as a product of activated T lymphocytes that inhibited the random migration of cultured macrophages [38]. MIF is also a key regulator of immune and inflammatory responses and is produced by a range of cells and tissues [39]. MIF expression can increase during the evolution of several malignances [40]. MIF has been implicated in the angiogenic switch during progression of early cancers and plays a role in macrophage-induced ovarian cancer cell invasiveness [41,42]. Furthermore, Serum levels of MIF have been used as part of algorithms to detect disease in women with BRCA1/2 mutations and for distinguishing malignant from benign disease [43].

IL-8, also known as C-X-C motif ligand (CXCL) 8 (CXCL8), is a small soluble protein that belongs to the CXC chemokine family [44]. Originally identified as a potent neutrophil activator and chemotactic factor, IL-8 is secreted by activated monocytes and macrophages and is dysregulated in a wide variety of solid cancers including ovarian cancer [45]. It is reported to contribute to multiple hallmarks of cancer, including increased proliferation, angiogenesis, invasion, and metastases [46-48]. While circulating IL-8 was not found to be a useful biomarker for early stage ovarian cancer, anti-IL-8 AAb detected at least one cancer missed by the other three markers in early and late stage. Why AAb develop against IL-8 is not well understood.

Previously, we reported that a four-biomarker panel comprising CA125, HE4, CA72-4 and MMP-7 showed strong diagnostic performance and significant improvement over the use of CA125 alone in distinguishing patients with early stage ovarian cancer from healthy women [49]. In subsequent studies, MMP-7 failed to detect cases missed by CA125, whereas HE4 and CA72.4 detected $16 \%$ of cases missed by CA125 in the UKCTOCS study [9]. The value of HE4 and CA72.4 were confirmed in the European Prospective Investigation into Cancer (EPIC) study [50]. Using a Luminex based assay, however, levels of HE4 were elevated in only $12 \%$ of early stage ovarian cancers and $36 \%$ of advanced stage disease. Moreover, the same cases could be identified with OPN, MIF and anti-IL-8 $\mathrm{AAb}$. Among the three additional biomarkers evaluated in this report, OPN was the most robust for detecting early stage disease in cases missed by CA125. Future studies will evaluate potential integration of OPN with CA125, HE4 and CA72-4.

\section{Materials and Methods}

\subsection{Patient Serum Samples}

A discovery set of preoperative sera from 76 patients with stage I-II and 44 patients with stage III-IV invasive epithelial ovarian cancer were obtained from the MD Anderson Gynecologic Cancer Tissue Bank. Sera from 200 healthy controls who had participated in the NROSS were also analyzed. An independent validation set included preoperative sera from 71 patients with stage I-II ovarian cancer, 45 preoperative sera from patients with stage III-IV ovarian cancer that had been drawn, processed and frozen more recently than the discovery set and stored in the same bank. Additional sera from 131 healthy controls were obtained from participants in the NROSS. This study was approved by the University of Texas MD Anderson Cancer Center ethical committee on the date 02/11/2019, ethical code number (LAB02-188). All participants had provided consent for use of samples in ethically approved studies. 


\subsection{Immunoassay Components}

MagPlex microspheres, xMAP antibody coupling kits and reagents for the immunoassay were purchased from Luminex Corp. (Austin, TX, USA). Recombinant human IL-8 (CXCL8) was purchased from PeproTech (Rocky Hill, NJ, USA). Human MDM2 protein with a His-tag, human PLAT/TPA protein with 6His-tag, human MYC/c-myc protein and human TACSTD1/EPCAM protein with a His-tag were obtained from LifeSpan BioSciences, lnc. (Seattle, WA, USA). MYC/DDK-tagged human HOXA7 protein was obtained from Creative Biomart (Shirley, NY, USA). Human Enolase-1 protein (ENO1), Human Annexin A2 protein (ANXA2), human protein disulfide isomerase (PDI) with his tag, human heat shock $70 \mathrm{kDa}$ protein (HSPA5) with his tag, human heat shock $70 \mathrm{kDa}$ protein-8 (HSPA8) with his tag were purchased from ProSpec-Tany TechnoGene Ltd. (Ness-Ziona, Israel). Human Cathepsin-D protein (CTSD) was obtained from Enzo Life Sciences, lnc. (Farmingdale, NY, USA). Biotin-conjugated mouse anti-IL-8 antibody and R-phycoerythrin conjugated F (ab') 2-Donkey anti-Rabbit IgG antibody were obtained from Invitrogen (Frederick, MD, USA). Biotin-conjugated mouse anti-MDM2 antibody was purchased from R\&D Systems (Minneapolis, MN, USA). Biotin-conjugated rabbit anti-PLAT antibody was purchased from LifeSpan BioSciences, lnc. Monoclonal mouse anti-c-Myc antibody was obtained from Thermo Fisher Scientific (Rockford, IL, USA). Biotin-conjugated mouse anti-CTSD antibody, biotin-conjugated mouse anti-ANXA2 antibody and biotin-conjugated mouse anti-ENO1 antibody were obtained from Novus Biologicals, LLC (Centennial, CO, USA). Biotin-conjugated mouse anti-HSC70 antibody was purchased from Enzo Life Sciences, Inc. Biotin-conjugated mouse anti-His antibody was obtained from Abcam (Cambridge, MA, USA). Rabbit anti-Myc-Tag antibody was purchased from Cell Signal Technology (Boston, MA, USA). Biotin-conjugated goat anti-human IgG Fc $\gamma$ fragment and anti-mouse IgG antibody were obtained from were obtained from Jackson ImmunoResearch (West Grove, PA, USA). Streptavidin-R-phycoerythrin (SAPE) was purchased from Life Technologies (Carlsbad, CA, USA). R-phycoerythrin (PE) conjugated goat anti-human IgG antibody was purchased from Southern Biotech (Birmingham, AL, USA).

\subsection{Antigen Immunoassays}

Enzyme-linked immunosorbent assays (ELISA) for 10 potential ovarian cancer serum markers (OPN, MIF, Leptin, IL-6, IL-8, TNFa, FGF2, HE4, TGFa, and VEGF) were performed using MILLIPLEX MAP human circulating cancer biomarker magnetic bead panel kits purchased from MilliporeSigma (Burlington, MA, USA). The median fluorescent intensity (MFI) was measured by the Luminex MAGPIX system (Luminex Corp., Austin, TX, USA) with a minimum of 50 beads read per well. The data were acquired and analyzed by xPONENT software version 4.2 (Luminex Corp.)

\subsection{Autoantibody Immunoassays}

Immunoassays for detecting AAbs against 12 antigens (IL-8, MDM2, c-MYC, PLAT, EpCAM, HOXA7, ENO1, PDI, HSPA5, HSPA8, ANXA2, and cathepsin D) were developed using the MagPlex/xMAP technology (Luminex Corp.). In brief, protein antigens were coupled to one million microspheres with an XMAP antibody coupling kit as specified by the manufacturer. Coupling efficiency was confirmed based on the xMAP Cookbook, thirs version (Luminex Corp.). For each assay, a suspension of antigen-microspheres was prepared by diluting the coupled microsphere stocks (one million beads $/ \mathrm{mL}$ ) to a final concentration of 50 beads $/ \mu \mathrm{L}$ in phosphate buffered saline (PBS) (Corning. NY. US). Serum samples $(2 \mu \mathrm{L})$ for assay were diluted in $48 \mu \mathrm{L}$ PBS buffer. Serum samples were added to 96-well polystyrene microplates and incubated with protein-coupled microspheres for $1 \mathrm{~h}$ at room temperature with gentle shaking. Beads were washed twice with PBS with $0.05 \%$ Tween 20 (Acros Organics, Geel, Belgium) (PBST) by clipping onto a magnetic plate separator for one minute and the liquid was discarded. Microspheres were then incubated with biotinylated goat anti-human IgG for $30 \mathrm{~min}$ at room temperature protecting from light with gentle shaking. Plates were washed twice with a PBST buffer and microspheres were incubated with a fluorescence reporter SAPE for $30 \mathrm{~min}$ 
at room temperature, protecting from light with gentle shaking. After a final wash, the beads were re-suspended in a PBS buffer and fluorescence measured on the MAGPIX system with a minimum 50 beads read per well. The data were acquired and analyzed by the xPONENT software version 4.2.

For anti-IL8 assays, conditions were further optimized. A suspension of IL-8 antigen-microspheres was diluted to a final concentration of 50 beads $/ \mu \mathrm{L}$ in assay buffer $(\mathrm{AB})$, which contained $1 \%$ bovine serum albumin (BSA) and 0.05\% Tween 20 in PBS. Serum samples ( $2 \mu \mathrm{L})$ for assay were diluted in $198 \mu \mathrm{L} \mathrm{AB}$, from which was transferred $25 \mu \mathrm{L}$ diluted sample to 96-well polystyrene microplates and incubated with $25 \mu \mathrm{L}$ IL-8-coupled microspheres for $2 \mathrm{~h}$ at room temperature with gentle shaking. Beads were washed twice with $\mathrm{AB}$ by clipping onto a magnetic plate separator for one minute and discarding the liquid. Microspheres were then incubated with $25 \mu \mathrm{L}$ goat anti-human IgG antibody with PE ( $4 \mathrm{ug} / \mathrm{mL}$ ) for $30 \mathrm{~min}$ at room temperature protecting from light with gentle shaking. After a final wash, the beads were re-suspended in AB and fluorescence measured on the MAGPIX system with a minimum of 50 beads read per well. The data were acquired and analyzed by xPONENT software version 4.2 .

\subsection{CA125 Immunoassay}

CA125 levels were assayed with Roche kits on the Elecsys platform (Indianapolis, IN, USA).

\subsection{Statistical Analysis}

Analysis was performed separately for early and late stage data sets. CA125 detected ovarian cancer cases with a cut-off of $35 \mathrm{U} / \mathrm{mL}$. Other biomarkers were dichotomized based on cut-offs that achieved $98 \%$ specificity. Logistic regression models were used to assess the discrimination of combined markers and to assess if adding new biomarkers can have additional benefit to discriminate cases and controls. AUC, sensitivity at specificity of $98 \%$, and partial AUC (pAUC) were calculated for each logistic model. Bootstrapping with 1000 bootstrap samples was used to compute $95 \%$ confidence intervals for the AUC and pAUC, as well as P values for testing the differences in these values. All statistical analyses performed using R software version 3.5.1 (R Foundation for Statistical Computing, Vienna, Austria).

\section{Conclusions}

Our study demonstrated that OPN, MIF and IL8 AAb can enhance the sensitivity of CA125 to distinguish ovarian cancer patients from health controls at high specificity. The development of reliable panels of tests that can detect cancer at an early and curable stage is worth further investigation.

Supplementary Materials: The following are available online at http://www.mdpi.com/2072-6694/11/5/596/s1, Figure S1: Serum levels of OPN, MIF, HE4 and IL-6 are elevated in the discovery set from ovarian cancer patients and healthy controls, Figure S2: Serum levels of Leptin, TNF $\alpha$, IL- 8 and VEGF are elevated in the discovery set from ovarian cancer patients and healthy controls, Figure S3: Serum levels of TGF- $\beta$, FGF2 are elevated in the discovery set from ovarian cancer patients and healthy controls, Figure S4: Serum autoantibody titers (AAb) of IL-8, PDI, HSPA8 and PLAT are elevated in the discovery set from ovarian cancer patients and healthy controls, Figure S5: Serum autoantibody titers (AAb) of HOXA7, ANX2, CTD and ENO1 are elevated in the discovery set from ovarian cancer patients and healthy controls, Figure S6: Serum autoantibody titers (AAb) of MDM2, Epcam, c-myc and HSPA5 are elevated in the discovery set from ovarian cancer patients and healthy controls, Table S1: Screening results of 22 candidates in discovery sample set.

Author Contributions: Conceptualization, R.C.B.J., Z.L. and J.G.; data curation, J.G., W.-L.Y. and J.C.; formal analysis, D.P. and J.N.; funding acquisition, R.C.B.J.; methodology, J.G. and W.-L.Y.; project administration, R.C.B.J. and Z.L.; supervision, R.C.B.J. and Z.L.; writing-original draft, J.G.; writing-review and editing, R.C.B.J., Z.L., K.H.L., A.E.L., Z.C.

Funding: This project was supported by grants from the National Cancer Institute Early Detection Research Network (5 U01 CA200462-02) (R.C.B.) and the MD Anderson Ovarian SPOREs (P50 CA83639 and P50CA217685) (R.C.B.), National Cancer Institute, Department of Health and Human Services; the Cancer Prevention Research Institute of Texas (RP160145) (R.C.B.); Minnesota Ovarian Cancer Alliance (R.C.B.), Golfer's Against Cancer, the Mossy Foundation, the Roberson Endowment, National Foundation for Cancer Research, UT MD Anderson Women's Moon Shot, and generous donations from Stuart and Gaye Lynn Zarrow. 
Conflicts of Interest: Dr. Bast receives royalties from Fujirebio Diagnostics Inc. for the discovery of CA125. The other authors declare no conflict of interest.

\section{References}

1. Key Statistics for Ovarian Cancer. Available online: https://www.cancer.org/cancer/ovarian-cancer/about/ key-statistics.html (accessed on 8 January 2019).

2. Survival Rates for Ovarian Cancer, by Stage. Available online: https:/www.cancer.org/cancer/ovariancancer/detection-diagnosis-staging/survival-rates.html (accessed on 11 April 2018).

3. Buys, S.S.; Partridge, E.; Greene, M.H.; Prorok, P.C.; Reding, D.; Riley, T.L.; Hartge, P.; Fagerstrom, R.M.; Ragard, L.R.; Chia, D.; et al. Ovarian cancer screening in the Prostate, Lung, Colorectal and Ovarian (PLCO) cancer screening trial: Findings from the initial screen of a randomized trial. Am. J. Obstet. Gynecol. 2005, 193, 1630-1639. [CrossRef] [PubMed]

4. Yang, W.L.; Gentry-Maharaj, A.; Simmons, A.; Ryan, A.; Fourkala, E.O.; Lu, Z.; Baggerly, K.A.; Zhao, Y.; Lu, K.H.; Bowtell, D.; et al. Elevation of TP53 autoantibody before CA125 in preclinical invasive epithelial ovarian cancer. Clin. Cancer Res. 2017, 23, 5912-5922. [CrossRef]

5. Fortner, R.T.; Damms-Machado, A.; Kaaks, R. Systematic review: Tumor-associated antigen autoantibodies and ovarian cancer early detection. Gynecol. Oncol. 2017, 147, 465-480. [CrossRef]

6. Lokshin, A.E.; Winans, M.; Landsittel, D.; Marrangoni, A.M.; Velikokhatnaya, L.; Modugno, F.; Nolen, B.M.; Gorelik, E. Circulating IL-8 and anti-IL-8 autoantibody in patients with ovarian cancer. Gynecol. Oncol. 2006, 102, 244-251. [CrossRef]

7. Skates, S.J.; Horick, N.; Yu, Y.; Xu, F.J.; Berchuck, A.; Havrilesky, L.J.; de Bruijn, H.W.; van der Zee, A.G.; Woolas, R.P.; Jacobs, I.J.; et al. Preoperative sensitivity and specificity for early-stage ovarian cancer when combining cancer antigen CA-125II, CA 15-3, CA 72-4, and macrophage colony-stimulating factor using mixtures of multivariate normal distributions. J. Clin. Oncol. 2004, 22, 4059-4066. [CrossRef]

8. Yurkovetsky, Z.; Skates, S.; Lomakin, A.; Nolen, B.; Pulsipher, T.; Modugno, F.; Marks, J.; Godwin, A.; Gorelik, E.; Jacobs, I.; et al. Development of a multimarker assay for early detection of ovarian cancer. J. Clin. Oncol. 2010, 28, 2159-2166. [CrossRef] [PubMed]

9. Bast, R.C.; Raamanathan, A.; Gentry-Maharaj, A.; Skates, S.; Baggerly, K.A.; Fourkala, R.; Ryan, A.; Zhang, Z.; Menon, U.; Jacobs, I. Validation of a multi-marker panel for early detection of ovarian cancer. J. Clin. Oncol. 2016, 34, 5570-5570. [CrossRef]

10. Holdenrieder, S.; Pagliaro, L.; Morgenstern, D.; Dayyani, F. Clinically meaningful use of blood tumor markers in oncology. Biomed. Res. Int. 2016, 2016, 9795269. [CrossRef]

11. Dudas, S.P.; Chatterjee, M.; Tainsky, M.A. Usage of cancer associated autoantibodies in the detection of disease. Cancer Biomark. 2010, 6, 257-270. [CrossRef] [PubMed]

12. Lu, D.; Kuhn, E.; Bristow, R.E.; Giuntoli, R.L., 2nd; Kjaer, S.K.; Shih Ie, M.; Roden, R.B. Comparison of candidate serologic markers for type I and type II ovarian cancer. Gynecol. Oncol. 2011, 122, 560-566. [CrossRef]

13. Agarwal, R.; Whang, D.H.; Alvero, A.B.; Visintin, I.; Lai, Y.; Segal, E.A.; Schwartz, P.; Ward, D.; Rutherford, T.; Mor, G. Macrophage migration inhibitory factor expression in ovarian cancer. Am. J. Obstet. Gynecol. 2007, 196, 348.e1-348.e5. [CrossRef]

14. Wei, R.; Wong, J.P.C.; Kwok, H.F. Osteopontin-A promising biomarker for cancer therapy. J. Cancer 2017, 8, 2173-2183. [CrossRef] [PubMed]

15. Drapkin, R.; von Horsten, H.H.; Lin, Y.; Mok, S.C.; Crum, C.P.; Welch, W.R.; Hecht, J.L. Human epididymis protein 4 (HE4) is a secreted glycoprotein that is overexpressed by serous and endometrioid ovarian carcinomas. Cancer Res. 2005, 65, 2162-2169. [CrossRef] [PubMed]

16. Ghasemi, A.; Hashemy, S.I.; Aghaei, M.; Panjehpour, M. RhoA/ROCK pathway mediates leptin-induced uPA expression to promote cell invasion in ovarian cancer cells. Cell Signal. 2017, 32, 104-114. [CrossRef] [PubMed]

17. Browning, L.; Patel, M.R.; Horvath, E.B.; Tawara, K.; Jorcyk, C.L. IL-6 and ovarian cancer: Inflammatory cytokines in promotion of metastasis. Cancer Manag. Res. 2018, 10, 6685-6693. [CrossRef] [PubMed] 
18. Le Page, C.; Ouellet, V.; Madore, J.; Hudson, T.J.; Tonin, P.N.; Provencher, D.M.; Mes-Masson, A.M. From gene profiling to diagnostic markers: IL-18 and FGF-2 complement CA125 as serum-based markers in epithelial ovarian cancer. Int. J. Cancer 2006, 118, 1750-1758. [CrossRef] [PubMed]

19. Buckanovich, R.J.; Sasaroli, D.; O’Brien-Jenkins, A.; Botbyl, J.; Hammond, R.; Katsaros, D.; Sandaltzopoulos, R.; Liotta, L.A.; Gimotty, P.A.; Coukos, G. Tumor vascular proteins as biomarkers in ovarian cancer. J. Clin. Oncol. 2007, 25, 852-861. [CrossRef] [PubMed]

20. Tiper, I.V.; Temkin, S.M.; Spiegel, S.; Goldblum, S.E.; Giuntoli, R.L., 2nd; Oelke, M.; Schneck, J.P.; Webb, T.J. VEGF potentiates GD3-mediated immunosuppression by human ovarian cancer cells. Clin. Cancer Res. 2016, 22, 4249-4258. [CrossRef]

21. Lau, T.S.; Chan, L.K.; Wong, E.C.; Hui, C.W.; Sneddon, K.; Cheung, T.H.; Yim, S.F.; Lee, J.H.; Yeung, C.S.; Chung, T.K.; et al. A loop of cancer-stroma-cancer interaction promotes peritoneal metastasis of ovarian cancer via TNFalpha-TGFalpha-EGFR. Oncogene 2017, 36, 3576-3587. [CrossRef]

22. Naora, H.; Montz, F.J.; Chai, C.Y.; Roden, R.B. Aberrant expression of homeobox gene HOXA7 is associated with mullerian-like differentiation of epithelial ovarian tumors and the generation of a specific autologous antibody response. Proc. Natl. Acad. Sci. USA 2001, 98, 15209-15214. [CrossRef]

23. Dai, L.; Li, J.; Ortega, R.; Qian, W.; Casiano, C.A.; Zhang, J.Y. Preferential autoimmune response in prostate cancer to cyclin B1 in a panel of tumor-associated antigens. J. Immunol. Res. 2014, 2014, 827827. [CrossRef]

24. Kim, J.H.; Herlyn, D.; Wong, K.K.; Park, D.C.; Schorge, J.O.; Lu, K.H.; Skates, S.J.; Cramer, D.W.; Berkowitz, R.S.; Mok, S.C. Identification of epithelial cell adhesion molecule autoantibody in patients with ovarian cancer. Clin. Cancer Res. 2003, 9, 4782-4791.

25. Zhang, J.Y.; Megliorino, R.; Peng, X.X.; Tan, E.M.; Chen, Y.; Chan, E.K. Antibody detection using tumor-associated antigen mini-array in immunodiagnosing human hepatocellular carcinoma. J. Hepatol. 2007, 46, 107-114. [CrossRef]

26. Sun, H.; Shi, J.X.; Zhang, H.F.; Xing, M.T.; Li, P.; Dai, L.P.; Luo, C.L.; Wang, X.; Wang, P.; Ye, H.; et al. Serum autoantibodies against a panel of 15 tumor-associated antigens in the detection of ovarian cancer. Tumour Biol. 2017, 39. [CrossRef]

27. Roberson, C.D.; Gercel-Taylor, C.; Qi, Y.; Schey, K.L.; Taylor, D.D. Identification of immunoreactive tumour antigens using free and exosome-associated humoral responses. J. Circ. Biomark. 2013, 1. [CrossRef]

28. Miller, D.M.; Thomas, S.D.; Islam, A.; Muench, D.; Sedoris, K. c-Myc and cancer metabolism. Clin. Cancer Res. 2012, 18, 5546-5553. [CrossRef]

29. Jacobs, I.J.; Menon, U. Progress and challenges in screening for early detection of ovarian cancer. Mol. Cell. Proteom. 2004, 3, 355-366. [CrossRef]

30. Menon, U.; Griffin, M.; Gentry-Maharaj, A. Ovarian cancer screening-Current status, future directions. Gynecol. Oncol. 2014, 132, 490-495. [CrossRef]

31. Schorge, J.O.; Drake, R.D.; Lee, H.; Skates, S.J.; Rajanbabu, R.; Miller, D.S.; Kim, J.H.; Cramer, D.W.; Berkowitz, R.S.; Mok, S.C. Osteopontin as an adjunct to CA125 in detecting recurrent ovarian cancer. Clin. Cancer Res. 2004, 10, 3474-3478. [CrossRef]

32. Rosen, D.G.; Wang, L.; Atkinson, J.N.; Yu, Y.; Lu, K.H.; Diamandis, E.P.; Hellstrom, I.; Mok, S.C.; Liu, J.; Bast, R.C., Jr. Potential markers that complement expression of CA125 in epithelial ovarian cancer. Gynecol. Oncol. 2005, 99, 267-277. [CrossRef]

33. Ballehaninna, U.K.; Chamberlain, R.S. Biomarkers for pancreatic cancer: Promising new markers and options beyond CA 19-9. Tumour Biol. 2013, 34, 3279-3292. [CrossRef] [PubMed]

34. Matsuura, M.; Suzuki, T.; Saito, T. Osteopontin is a new target molecule for ovarian clear cell carcinoma therapy. Cancer Sci. 2010, 101, 1828-1833. [CrossRef] [PubMed]

35. Song, G.; Cai, Q.F.; Mao, Y.B.; Ming, Y.L.; Bao, S.D.; Ouyang, G.L. Osteopontin promotes ovarian cancer progression and cell survival and increases HIF-1alpha expression through the PI3-K/Akt pathway. Cancer Sci. 2008, 99, 1901-1907. [CrossRef]

36. Zhao, H.; Chen, Q.; Alam, A.; Cui, J.; Suen, K.C.; Soo, A.P.; Eguchi, S.; Gu, J.; Ma, D. The role of osteopontin in the progression of solid organ tumour. Cell Death Dis. 2018, 9, 356. [CrossRef]

37. Zhang, L.L.; Shao, S.L.; Wu, Y. Expressions of osteopontin and B7-H4 in epithelial ovarian neoplasm and their significance. Chin. J. Cancer 2010, 29, 25-29. [CrossRef] 
38. Bacher, M.; Metz, C.N.; Calandra, T.; Mayer, K.; Chesney, J.; Lohoff, M.; Gemsa, D.; Donnelly, T.; Bucala, R. An essential regulatory role for macrophage migration inhibitory factor in T-cell activation. Proc. Natl. Acad Sci. USA 1996, 93, 7849-7854. [CrossRef]

39. Calandra, T.; Roger, T. Macrophage migration inhibitory factor: A regulator of innate immunity. Nat. Rev. Immunol. 2003, 3, 791-800. [CrossRef]

40. Mitchell, R.A. Mechanisms and effectors of MIF-dependent promotion of tumourigenesis. Cell Signal. 2004, 16, 13-19. [CrossRef]

41. Wilson, J.M.; Coletta, P.L.; Cuthbert, R.J.; Scott, N.; MacLennan, K.; Hawcroft, G.; Leng, L.; Lubetsky, J.B.; Jin, K.K.; Lolis, E.; et al. Macrophage migration inhibitory factor promotes intestinal tumorigenesis. Gastroenterology 2005, 129, 1485-1503. [CrossRef]

42. Hagemann, T.; Wilson, J.; Kulbe, H.; Li, N.F.; Leinster, D.A.; Charles, K.; Klemm, F.; Pukrop, T.; Binder, C.; Balkwill, F.R. Macrophages induce invasiveness of epithelial cancer cells via NF-kappa B and JNK. J. Immunol. 2005, 175, 1197-1205. [CrossRef]

43. Gschwantler-Kaulich, D.; Weingartshofer, S.; Rappaport-Furhauser, C.; Zeillinger, R.; Pils, D.; Muhr, D.; Braicu, E.I.; Kastner, M.T.; Tan, Y.Y.; Semmler, L.; et al. Diagnostic markers for the detection of ovarian cancer in BRCA1 mutation carriers. PLOS ONE 2017, 12, e0189641. [CrossRef] [PubMed]

44. Rossi, D.; Zlotnik, A. The biology of chemokines and their receptors. Annu. Rev. Immunol. 2000, 18, $217-242$. [CrossRef]

45. Yoshimura, T.; Matsushima, K.; Tanaka, S.; Robinson, E.A.; Appella, E.; Oppenheim, J.J.; Leonard, E.J. Purification of a human monocyte-derived neutrophil chemotactic factor that has peptide sequence similarity to other host defense cytokines. Proc. Natl. Acad. Sci. USA 1987, 84, 9233-9237. [CrossRef] [PubMed]

46. Singh, S.; Sadanandam, A.; Nannuru, K.C.; Varney, M.L.; Mayer-Ezell, R.; Bond, R.; Singh, R.K. Small-molecule antagonists for CXCR2 and CXCR1 inhibit human melanoma growth by decreasing tumor cell proliferation, survival, and angiogenesis. Clin. Cancer Res. 2009, 15, 2380-2386. [CrossRef] [PubMed]

47. Seaton, A.; Scullin, P.; Maxwell, P.J.; Wilson, C.; Pettigrew, J.; Gallagher, R.; O'Sullivan, J.M.; Johnston, P.G.; Waugh, D.J. Interleukin-8 signaling promotes androgen-independent proliferation of prostate cancer cells via induction of androgen receptor expression and activation. Carcinogenesis 2008, 29, 1148-1156. [CrossRef]

48. Zhu, Y.M.; Webster, S.J.; Flower, D.; Woll, P.J. Interleukin-8/CXCL8 is a growth factor for human lung cancer cells. Br. J. Cancer 2004, 91, 1970-1976. [CrossRef]

49. Simmons, A.R.; Clarke, C.H.; Badgwell, D.B.; Lu, Z.; Sokoll, L.J.; Lu, K.H.; Zhang, Z.; Bast, R.C., Jr.; Skates, S.J. Validation of a biomarker panel and longitudinal biomarker performance for early detection of ovarian cancer. Int. J. Gynecol. Cancer 2016, 26, 1070-1077. [CrossRef]

50. Terry, K.L.; Schock, H.; Fortner, R.T.; Husing, A.; Fichorova, R.N.; Yamamoto, H.S.; Vitonis, A.F.; Johnson, T.; Overvad, K.; Tjonneland, A.; et al. A prospective evaluation of early detection biomarkers for ovarian cancer in the European EPIC cohort. Clin. Cancer Res. 2016, 22, 4664-4675. [CrossRef] 